\title{
El manejo agrícola como herramienta clave para una agricultura de conservación. Su análisis desde la bioquímica y la microbiología del suelo.
}

\section{The agricultural management as a key tool for a conservative agriculture. Its analysis through biochemistry and microbiology.}

\author{
Juan Pablo Frene \\ Universidad Nacional de Quilmes, Laboratorio de Bilogía de Suelos, Argentina. \\ Contacto: juanpifrene@hotmail.com
}

\section{Luciano Andrés Gabbarini}

Universidad Nacional de Quilmes, Argentina.

Contacto: luciano.gabbarini@gmail.com

\author{
Luis Gabriel Wall \\ Universidad Nacional de Quilmes, Argentina. \\ Contacto: wall.luisgabriel@gmail.com
}

Recibido: marzo de 2018

Aceptado: mayo de 2018

\begin{abstract}
Resumen
Los nuevos desafíos mundiales producto del crecimiento demográfico ponen en evidencia la necesidad de llevar a cabo cambios en las formas de cultivar el suelo, para lo cual es necesario modificar las prácticas agrícolas actuales. En este trabajo nos proponemos hacer foco en cómo los manejos agrícolas influencian y alteran las características del suelo y los servicios ecosistémicos del mismo. Aquí presentamos evidencias de los efectos benéficos producidos por la siembra directa sobre las propiedades físicas, químicas y biológicas del suelo. Posteriormente discutimos qué sucede cuando los manejos agrícolas son intercambiados y el suelo debe adaptarse a una nueva situación. Finalmente concluimos con lo que sucede cuando se cambia el enfoque y se analiza el suelo cuando es separado en las subestructuras de agregados que lo forman. Además, qué influencias tienen los manejos agrícolas sobre las diferentes fracciones de agregados.
\end{abstract}

Palabras clave: bioquímica de suelos; microbiología de suelos;agricultura de conservación; siembra directa; labranza convencional. 
Abstract: The new global challenges show the necessity of carry out changes in the ways of soil agricultural management, for which it is necessary to modify the current agricultural practices. In this review, we focus on how agricultural management influence and alter the characteristics of the soil and its ecosystem services. Here, we present evidence of the beneficial effects produced by no-tillage on physical, chemical and biological properties of the soil. Later, we discuss what happens when the agricultural management is exchanged and the soil must adapt to a new situation. Finally, we conclude with what happens when the focus is changed and the soil is analyzed when it is separated in its aggregates that form it. In addition, we analyzed what influences have the agricultural management on different soil aggregates fractions.

Keywords: soil biochemistry; soil microbiology; conservative agriculture;no-tillage;conventional tillage.

\subsection{Introducción: El suelo como sistema.}

El suelo es un sistema vivo y complejo, es uno de los principales reservorios biológicos del planeta donde se lleva a cabo gran parte del ciclo de los elementos químicos y la transformación de la materia. A partir del suelo la sociedad obtiene gran parte de los servicios ecosistémicos, los cuales son considerados beneficiosos, como lo es el proveer alimento y energía, regular la calidad del agua y soportar el ciclado de los nutrientes (Millenium Ecosystem Assessment, 2005). Los servicios ecosistémicos sientan sus bases en los organismos vivos que habitan el suelo, desde las plantas y los animales pasando por la macro y microfauna hasta las comunidades microbianas. Los diferentes manejos y usos del suelo modifican las características químicas, físicas y biológicas del mismo, condicionando así la estructura de las comunidades microbianas que lo habitan. Como consecuencia del constante aumento de la población mundial, y con ello la creciente demanda de alimentos, es esencial poder incrementar los servicios ecosistémicos provenientes del suelo y la agricultura con el fin de suplir esta demanda evitando, a su vez, generar degradación y pérdida del suelo (Foley, 2011).

Una forma de incrementar las prestaciones ecosistémicas es a través de la agricultura de conservación (Albertengo et al., 2013). Dentro de este tipo de manejo se encuentra la siembra directa, como un componente importante dentro de los sistemas agrícolas como también lo son la rotación y diversificación de cultivos y el uso de cultivos de cobertura en invierno. La siembra directa es utilizada y considerada en la agricultura en contraposición a la labranza convencional, ya que esta última produce la erosión y la disminución de la salud del suelo (Triplett \& Dick, 2008).

Los desafíos actuales como la pérdida de la calidad de los suelos, la necesidad de producir más y de mejor forma, exacerbado por el cambio climático, plantean el requisito de llevar a cabo cambios en la manera de hacer producir al suelo. El desarrollo del conocimiento científico y la creciente conciencia de la humanidad acerca del suelo como recurso no renovable están cambiando el paradigma de la agricultura de un modelo químico a uno biológico, es decir, el reemplazo de los productos químicos, como los 
fertilizantes, por productos biológicos, como los inoculantes. En este trabajo nos proponemos evaluar los efectos producidos en los agroecosistemas cuando los mismos son afectados por cambios en sus manejos agrícolas. De esta manera, se compara cómo las prácticas agrícolas -esto es, la siembra directa (SD) y la labranza convencional (LC)modifican los diferentes parámetros del suelo. Posteriormente, se analiza la dinámica de cambio de parámetros bioquímicos y microbiológicos cuando en los suelos se produce el cambio de un manejo al otro, incluyendo un análisis a nivel de los microagregados de suelo.

\subsection{Prestaciones funcionales del suelo}

Los servicios ecosistémicos y los factores bióticos del suelo están determinados en gran proporción por factores abióticos, entre los que podemos incluir a las propiedades físicas y químicas del suelo así como también a los factores climáticos y edáficos propios de cada región. Dentro de los factores bióticos podemos observar a las plantas, a la fauna y a los microorganismos del suelo. Esta última se encuentra compuesta principalmente por bacterias, hongos y arqueas, pudiendo llegar a representar cerca del $80 \%$ de la biomasa del suelo (Nannipieri et al., 2003). Los microorganismos son los agentes primarios del cambio debido a sus capacidades de liberar enzimas extracelulares, lo que los hace únicos para llevar a cabo los procesos biogeoquímicos. Su diversidad nutricional y la capacidad de liberar una gran variedad de enzimas distintas los posiciona como un engranaje fundamental como agentes direccionadores de los ciclos de los nutrientes (Madsen, 2005). Las enzimas, liberadas tanto por los microorganismos como por las plantas, catalizan la ruptura de moléculas complejas y hacen disponibles los nutrientes para los habitantes del suelo. Las actividades enzimáticas responden a la disponibilidad de los sustratos, a la demanda de los nutrientes, a la actividad microbiana y a las condiciones ambientales (Allison et al., 2010).

El principal factor abiótico que regula la actividad microbiana es la disponibilidad de las fuentes de energía, siendo el carbono el nutriente más abundante (Finn et al., 2017). Por ello, los cambios en la disponibilidad y en la calidad del carbono tienen un profundo efecto en los procesos biológicos. Por ejemplo, la etapa inicial de la asimilación microbiana del carbono orgánico por parte de los microorganismos, depende fuertemente del tipo y la calidad de los residuos y restos de cultivos que quedan en el suelo. Esta composición determina la velocidad con que las enzimas son capaces de degradar las moléculas de celulosa -componente principal de la pared celular de las plantas- transformándolas en sustancias carbonadas disponibles para los microorganismos. El nitrógeno, al igual que el carbono, es un elemento vital para el desarrollo de los seres vivos; sin embargo, este se 
encuentra en cantidades limitantes en el suelo. Por lo cual, con el objetivo de incrementar el rendimiento de los cultivos, las prácticas agrícolas habituales agregan grandes cantidades de fertilizantes nitrogenados al suelo (Nelson et al., 2016).

Por otro lado, varias propiedades físicas del suelo son importantes en la regulación de la actividad microbiana. Podemos mencionar el tamaño, la distribución y la tasa de formación de los agregados, el tamaño y la red de poros y la mineralogía del suelo (Bronick \& Lal, 2005). Por ejemplo, los agregados de mayor tamaño (macroagregados) tienen la capacidad de almacenar y estabilizar materia orgánica en su interior, la cual queda protegida de la degradación por parte de los microorganismos (Balesdent et al., 2000). Los manejos agrícolas como la labranza del suelo producen una disminución significativa de los macroagregados, pudiendo producir una pérdida del carbono por posterior erosión (Hutchinson et al., 2007) que vuelve como dióxido de carbono a la atmósfera aumentando el efecto invernadero.

\subsection{Los manejos agrícolas}

La historia de la labranza tiene sus comienzos miles de años atrás cuando los seres humanos cambiaron los hábitos nómades de la caza y la recolección, por formas de vida sedentarios, empezaron a cultivar la tierra y a utilizar la labranza para mejorar la germinación y en tanto técnica de control de malezas. En el siglo XIX, con la llegada de la Revolución Industrial, el poder mecánico generado por los primeros motores y los tractores facilitaron la labranza del suelo. La labranza convencional (LC) es una práctica en la cual se trazan surcos de una determinada profundidad en la superficie del suelo, a partir de la cual, pierde su estructura original y se mezcla. La labranza tiene dos objetivos principales: por un lado el control de la estructura para beneficiar a las semillas durante la siembra, y por el otro lado, la incorporación de los residuos orgánicos en el suelo y su posterior mineralización (Balesdent et al., 2000). La agricultura basada en la labranza ha exacerbado los problemas de la degradación del suelo por efecto del agua y el viento, la oxidación de la materia orgánica y una disminución de la capa cultivable y de la estructura del suelo en ciertos ambientes (Six et al., 1999).

En la búsqueda de una alternativa y solución a los efectos negativos producidos por la LC, a mediados del siglo pasado, se desarrolló una agricultura sin laboreo con la posibilidad de disminuir el impacto sobre el suelo y el ambiente. La siembra directa (SD) surgió en respuesta a los problemas de erosión producidos por la LC y continuó popularizándose a gran escala durante la década del 40 y del 50 en los Estados Unidos. En la SD, el suelo permanece completamente imperturbable durante la siembra excepto por el pequeño surco producido para depositar la semilla y donde el control de las malezas se hace por medio de herbicidas y otros cultivos (Albertengo et al., 2013). La SD ha sido 
reconocida como una práctica efectiva para conservar y mejorar la salud del suelo (Lal, 2004). Al comparar la SD con la LC se observa que reduce la erosión y la pérdida del suelo, incrementa la materia orgánica y el carbono almacenado producto de los restos de los cultivos que quedan en la superficie (West \& Post, 2002; Pandey et al., 2014), mejorando, a su vez, las propiedades físicas como el aumento de la capacidad de retención de agua y la reducción de la compactación del suelo (Derpsch et al., 2010). A estos beneficios se suma los menores costos que tiene la SD en término de combustible y en razón del arado que no realiza (Lal, 2004). Estudios realizados por diversos laboratorios incluido el nuestro (Laboratorio de Biología de Suelos-UNQ) muestran que la SD presenta mayores valores de carbono, fósforo y biomasa microbiana en comparación con la LC. A nivel físico, la SD presenta mayores valores de agregados de tipo macroagregados, en cambio, los agregados de menor tamaño resultan más abundantes en la LC. En consonancia con estos resultados, gran variedad de estudios han mostrado que la labranza rompe los macroagregados del suelo (Beare et al., 1994; Kandeler et al., 1999; Six et al., 2002; Jiang et al., 2011). Además, estos aspectos químicos y físicos posiblemente se encuentren relacionados, ya que los agregados de mayor tamaño ofrecen una mayor protección a la materia orgánica ocluida en su interior (Franzluebbers \& Arshad, 1996; Christensen, 2001).

A nivel bioquímico, la actividad enzimática se encuentra exacerbada en la SD, posiblemente atribuido a un mayor aporte de nutrientes por los rastrojos que quedan cubriendo la superficie del suelo (Roldán et al., 2005; Zhang et al., 2014) o a una mayor estabilidad de las enzimas adsorbidas en la matriz de los suelos debido a ser un ambiente menos oxidátivo producto de un menor disturbio y una menor disponibilidad de oxígeno (Acosta-Martínez et al., 2008). Por otro lado, la actividad microbiana, medida a través de curvas de consumo de oxígeno con diferentes fuentes de carbono adicionadas, posibilitó diferenciar a las muestras de la SD de aquellas con LC, presentando estas últimas una mayor actividad microbiana posiblemente a consecuencia de que la descomposición y mineralización se encuentren aceleradas (Gomez \& Garland, 2012).

Estudios de la estructura de la comunidad microbiana por medio del gen risobosomal 16S (Pirosecuenciación) muestran que es posible encontrar géneros de bacterias particulares promovidos por la SD o por la LC, como en el caso de las bacterias copiotróficas, como los Firmicutes y las $\beta$-Proteobacteria, que se encuentran en una mayor abundancia en los suelos en LC en comparación con los suelos en SD, donde sobresalen las Acidobacterias y los Verrumicrobios (Silva et al., 2013; Dai et al., 2017). Esto sugiere que los manejos agrícolas constituyen una fuerza impulsora lo suficientemente fuerte como para modificar parcialmente la diversidad bacteriana.

En un análisis de metadatos (donde se analizan muchos trabajos publicados por diferentes autores) llevado a cabo por Pittelkow y colaboradores (2015) se encontró que la 
SD per se tiene un impacto negativo en el rendimiento de los cultivos, con una disminución cercana al $6 \%$, principalmente en regiones húmedas del planeta donde la SD tiene menos beneficios debido a las mejores condiciones de humedad del suelo. El valor de la SD aparece cuando se combina con otros elementos que definen a la agricultura de conservación. Por lo tanto, es indispensable el uso del paquete completo que constituye la agricultura de conservación. Esto es posible de observar en otro estudio de este tipo donde se incluyen otros parámetros además del rendimiento de los cultivos y se aprecia los amplios beneficios de la SD a nivel físico, químico y biológico en términos de sustentabilidad del sistema (Zhao et al., 2017).

\subsection{Caso de Estudios: De la siembra directa a la labranza convencional, y viceversa.}

El cambio de la LC a la SD como sistema de cultivo tiene el propósito de evitar la degradación de los suelos y mejorar la salud del mismo. Además de aumentar el carbono almacenado en el suelo con el fin de disminuir el efecto invernadero (Crowther et al., 2016). Sin embargo, la transición de la LC a la SD no es inmediata. Se ha reportado que durante los primeros años, el suelo sufre un retardo en la respuesta frente a la SD en indicadores tales como un aumento del carbono almacenado en el suelo, y una disminución en el rendimiento de los cultivos y en la eficiencia en el ciclado de los nutrientes (Phatak et al., 1999), producto principalmente del nuevo estado del suelo.

Existen varios estudios sobre el pasaje de la SD a la LC a nivel del contenido de carbono orgánico del suelo (Franzluebbers \& Arshad, 1996; Díaz-Zorita et al., 2002; West \& Post, 2002; Alvarez \& Steinbach, 2009; Du et al., 2014) y a nivel de cambios en las comunidades microbianas por análisis de la composición de ácidos grasos de su pared celular (Feng et al., 2003; Simmons \& Coleman, 2008; Wortmann et al., 2008; Jiang et al., 2011b). Sin embargo, no se ha estudiado en profundidad lo que sucede con las comunidades microbianas del suelo a nivel bioquímico durante el cambio de sistema de labranza, en el traspaso de la SD a la LC, o viceversa, en una etapa temprana posterior al cambio. Entender los efectos durante el periodo de transición o adaptación al nuevo sistema agrícola es clave para un manejo efectivo del suelo y una correcta toma de decisiones por parte del agricultor. Un ensayo donde se produjo un cambio de labranza fue llevado a cabo por nuestro laboratorio a partir del cual se analizó la variación de las propiedades del suelo durante los primeros tres años. En este ensayo llevado a cabo en el sudoeste de la provincia de Buenos Aires, una parcela agrícola donde se lleva a cabo la LC, se dividió en dos a partir de la cual una parte pasó a SD (nueva SD) y la otra continúo en LC (control histórico). Lo mismo sucedió con otra parcela donde se realizaba la SD, quedando dividida en SD (control histórico) y otra parte pasó a LC (nueva LC). 
A nivel químico, los suelos que sufrieron un cambio en su manejo (independientemente de cuál sea su nuevo manejo) muestran que las concentraciones de carbono y fósforo disponible sufren una disminución posterior al cambio respecto a los controles y en los tres años que se desarrolló el ensayo no se alcanzó a observar una recuperación a niveles similares a los de los controles históricos. Esta disminución en ciertos parámetros químicos ha sido ampliamente descripta por otros autores (West \& Post, 2002; Lal, 2015). Lal (1998) establece que de los 2 a los 5 años posteriores al cambio de labranza puede ocurrir un leve o nulo aumento del carbono almacenado en el suelo y posteriormente, entre los 5 a los 10 años se verifica un gran aumento en la acumulación de carbono. Por otra parte, en nuestros ensayos los cambios físicos en la estructura del suelo se produjeron de manera más veloz al disminuir los agregados de mayor tamaño producto de la ruptura de los mismos con la LC (Beare et al., 1994). A nivel bioquímico es posible seguir la dinámica de cambio de los manejos agrícolas por medio de la actividad enzimática, donde las actividades en los suelos que comenzaron a tener SD fueron aumentando con el transcurso del tiempo, acercándose a valores similares a su control histórico (SD). A su vez, sucedió lo opuesto con LC y los suelos que comenzaron a tener LC. Al mostrar respuestas tan dinámicas, es posible que las actividades enzimáticas sean una buena elección como parámetro de respuesta a cambios de manejo para estudiar el suelo, ya que al parecer son mucho más sensibles que el resto de los indicadores de origen químico o físico, como es el carbono total o la materia orgánica, a los cambios de manejo. En esta misma línea de pensamiento, Kandeler y colaboradores (1999) observaron, en un estudio de largo plazo, que la actividad enzimática de la fosfatasa ácida era una de las mediciones que mostraban signos de cambio en el pasaje de la LC hacia la SD de forma temprana.

Cuando se analizó la estructura bacteriana por medio de herramientas moleculares a partir del ADN extraído directamente del suelo, los suelos que recientemente habían sufrido cambio en sus manejos mostraron un mapa de diversidad bacteriana diferente al de los controles históricos, posiblemente una diversidad microbiana relacionada con un estado de transición. Por su parte, los mapas de diversidad de los suelos con manejos históricos de SD o LC resultaron claramente diferentes entre sí. Encontramos una diferenciación en profundidad de la diversidad bacteriana en el caso de SD donde el suelo no se perturba, que no se observa en el caso de LC donde el suelo se mezcla regularmente homogenizando aparentemente las comunidades microbianas en la profundidad de la labranza. Feng y colaboradores (2003) encontraron cambios similares en la composición de las comunidades microbianas en suelos con LC y SD donde se cultivaba algodón. Sin embargo, otros estudios mostraron que los cambios por tipo de labranza son menos significativos que los efectos producidos por el tipo de cultivo y el tipo de residuo dejado por los mismos (Dıaz-Zorita et al., 2002). Esto permite distinguir dos 
tipos de efectos sobre el suelo a tener en cuenta, el efecto mecánico y el efecto de la planta.

\subsection{Estudio a nivel de los agregados del suelo.}

En los estudios del suelo en el cual se involucran microorganismos, en líneas generales, no se le pone demasiado énfasis a la escala espacial de trabajo. Comúnmente se trabaja con muestras de suelo homogenizadas que tratan de representar un lote. Es decir, por lo general se ha pensado en la escala humana y no en la escala microbiológica. Esta escala microbiana nos lleva a pensar en términos de los microagregados, que en su conjunto constituyen la estructura del suelo. La incorporación del concepto de estructura del suelo es esencial debido a las profundas influencias que tiene el hábitat microbiano y sus actividades en dirigir los ciclos del carbono y los elementos (nutrientes), como el nitrógeno y el fósforo, que luego se expresan a escala de paisaje en el suelo del ecosistema (Ettema \& Wardle, 2002; Philippot et al., 2009).

Los agregados del suelo constituyen un nicho ecológico donde sus propiedades físico-químicas seleccionarían la distribución de los microorganismos entre los diferentes tamaños de agregados (Mummey et al., 2006). La preferencia de un grupo microbiano por una locación específica en la matriz del suelo para crecer y utilizar substratos allí presentes puede estar relacionado con la accesibilidad de los mismos, pero también, y tal vez más importante, con las condiciones físico-químicas únicas en ese hábitat de suelo (Kong et al., 2011). Un análisis metagenómico, es decir, un estudio donde se analiza todo el ADN extraído del suelo, de la estructura microbiana encontró que aproximadamente la mitad de la variación en la composición de la comunidad microbiana puede ser explicada por la distancia espacial y los propiedades particulares del hábitat (Davinic et al., 2012). La distribución de la biomasa microbiana es heterogénea en el suelo, encontrándose la mayor abundancia de las bacterias y los hongos, así como también la mayor diversidad, en las fracciones de agregados más pequeñas (Bach et al., 2018). De esta forma se sabe que el suelo no es homogéneo, sino que cada fracción de agregados es un hábitat que ejerce una presión de selección diferente sobre los microorganismos que se encuentran allí presentes (Kandeler et al., 1999c; Neumann et al., 2013; Sessitsch et al., 2001; Vos et al., 2013).

A nivel funcional, se ha reportado un patrón de actividad microbiana que revela una mayor actividad específica en los agregados de menor tamaño (Hattori, 1988). Esto se correlaciona con la distribución de la biomasa microbiana y el carbono orgánico disponible en las diferentes fracciones de agregados (Lagomarsino et al., 2012). En nuestras investigaciones, la actividad microbiana analizada por medio de perfiles fisiológicos a nivel de comunidades en diferentes fracciones de agregados posibilitó la diferenciación de acuerdo al tamaño de las fracciones independientemente del manejo agrícola (Frene, 
2017). Esto se encuentra en sintonía con lo observado en la cuantificación de las abundancias de algunos grupos microbianos, ya que se cree que los perfiles fisiológicos son producidos por microorganismos copiotróficos presentes en las muestras de suelo (Lladó \& Baldrian, 2017).

La locación específica de las enzimas extracelulares dentro de la matriz del suelo ha sido especialmente estudiada en relación a la calidad de la materia orgánica de suelo (Kandeler et al., 1999a) y su recambio en el mismo (Stemmer et al., 1998). La predominancia de las actividades enzimáticas en las fracciones de agregados de suelo parece depender tanto de la locación de los microorganismos así como también de los mecanismos de unión y adsorción de las moléculas de enzimas a las partículas minerales u orgánicas (Kandeler et al., 1999b; Lagomarsino et al., 2012). Por el contrario, la distribución de las actividades enzimáticas del suelo en las fracciones de agregados presenta una distribución diferencial de acuerdo al manejo agrícola del suelo analizado. Posiblemente esto se deba a que la LC redistribuye el carbono a lo largo de todo el perfil del suelo, en cambio, en la SD el suelo se mantiene sin disturbios físicos y los depósitos de carbono más lábiles se encuentran en las fracciones de agregados más grandes, favoreciendo así la síntesis y excreción de enzimas (Tiemann et al., 2015; Tiemann \& Grandy, 2015; Trivedi et al., 2017).

Hasta el momento una gran cantidad de estudios nos presentan diferencias entre los suelos con SD y LC (Acosta-Martínez et al., 2008; Jangid et al., 2008; Kandeler et al., 1999a; Silva et al., 2013; Simmons \& Coleman, 2008). La SD aumenta la cantidad de macroagregados y el carbono almacenado en el suelo, favoreciendo así la actividad enzimática en comparación con la LC (Mbuthia et al., 2015). Cuando analizamos el cambio de manejo pudimos ver que la labranza produce la ruptura y la disminución de los macroagregados favoreciendo así la perdida de estructura del suelo. Además se produce una caída de la actividad enzimática hasta alcanzar valores similares a los observados en suelos con historia de LC de larga data. Cuando se lleva a cabo el pasaje hacia la SD se observa que los macroagregados tienden a aumentar así como también la actividad enzimática.

\subsection{Efecto del cambio de manejo agrícola a nivel de agregados del suelo.}

Hasta el momento se han presentado pocos estudios donde se analicen los manejos agrícolas como la SD o la LC a nivel de agregados, menos aún en aquellos que sufren un disturbio como puede ser el cambio de labranza del suelo (Gupta \& Germida, 1988; Kandeler et al., 1999b; Jiang et al., 2011b). Al analizar el cambio de manejo agrícola a nivel de las fracciones de agregados se observó un aumento de las fracciones de mayor tamaño en los suelos que pasan a SD, y en cambio, una disminución en aquellos suelos 
que cambian a LC respecto a sus manejos predecesores. Sucedió lo opuesto en las fracciones de menor tamaño (Helgason et al., 2010; Zhang et al., 2016). La actividad enzimática presentó una distribución de acuerdo al tamaño de las fracciones de agregados, encontrándose los mayores valores en las fracciones de menor tamaño. Esto se debe posiblemente a quelas actividades de las enzimas involucradas en el ciclado de los nutrientes son mayores en los agregados más pequeños, como consecuencia de que la concentración de carbono es mayor en estas fracciones de agregados comparados con agregados de mayor tamaño (Qin et al., 2010; Tiemann et al., 2015; Trivedi et al., 2015).

Cuando se analizó la estructura de las comunidades microbianas en las diferentes fracciones de agregados por medio de la cuantificación de los grupos principales (bacterias, hongos y arqueas) se observó una distribución de acuerdo al tamaño de fracción, similar a la observada en las actividades enzimáticas (Bach et al., 2018). Las mayores diferencias entre los tratamientos agrícolas se presentaron para los tres grupos microbianos en la fracción pequeña, donde sobresalieron los hongos no hubo diferencias entre el suelo que sufrió un cambio a LC y los suelos con LC por más de 30 años. Posiblemente esta última observación se deba a que los hongos son muy sensibles a la pérdida de estructura del suelo producto de la labranza, ya se produce la ruptura de sus hifas (Six et al., 2006; Wakelin et al., 2008).

Cuando se analizó solo la estructura bacteriana al cuantificar algunos de los principales filos y clases, como lo son Acidobacterias, Actinobacterias, $\alpha$-Proteobacteria, $\beta$ Proteobacteria y Firmicutes, se observó una adaptación a la hipótesis de estrategias de vida copiotróficos/oligotróficos entre las bacterias del suelo. Esta teoría ecológica presupone la existencia de organismos con la capacidad de crecer de manera rápida cuando hay una abundancia de nutrientes y reciben el nombre de organismos copiotróficos; otros organismos que pueden crecer cuando hay escasez de nutrientes y que reciben el nombre de organismos oligotróficos. En la fracciones de mayor tamaño los grupos copiotróficos ( $\alpha$-proteobacteria, $\beta$-proteobacteria y Firmicutes) presentaron las mayores abundancias en la LC, en cambio, los oligotróficos (Acidobacterias y Actinobacterias) lo hicieron en la nueva SD. Cuando analizamos la dinámica de cambio de manejo a través de las variaciones relativas de los parámetros respecto a los valores de dicho parámetro en el caso del tratamiento original histórico, vimos en las fracciones de mayor tamaño que con el cambio de manejo aumentan las bacterias oligotróficas mientras que las bacterias copiotróficas disminuyen. En las fracciones de menor tamaño, en cambio, los filos y clases bacterianos se comportan de manera opuesta de acuerdo al cambio de tratamiento que hayan sufrido, donde la nueva SD aumenta con respecto a tu tratamiento predecesor y la nueva LC disminuye respecto a la SD predecesora. Esto señala la sensibilidad de las diferentes fracciones frente a los cambios de manejos. Hasta el momento, los pocos trabajos publicados donde se estudian cambios que sufre el suelo a 
nivel de las fracciones de agregados muestran que las fracciones de macroagregados son más sensibles al cambio (Trivedi et al., 2017).

Estas observaciones sugieren que el ensamblaje de las comunidades microbianas del suelo es un balance que combina las influencias de los tamaños de agregados y los manejos agrícolas. En definitiva, los cambios en los manejos agrícolas tendrían efectos directos e indirectos sobre las comunidades microbianas, haciendo dificultoso de elucidar los mecanismos de respuesta de las comunidades microbianas (Leff et al., 2015).

Actualmente la cantidad de estudios científicos que adecuan la escala de estudio al separar el suelo en sus componentes, como lo son las fracciones de agregados, es cada vez mayor. Estudios recientes que analizan las comunidades microbianas en diferentes fracciones de agregados muestran que se presentan características heterogéneas de acuerdo a su tamaño, es decir, que cada fracción de agregados conforma un nicho ecológico diferencial con sus propias condiciones físico-químicas donde se favorecen comunidades microbianas con diferentes propiedades y composición estructural (Davinic et al., 2012; Constancias et al., 2014; Bach \& Hofmockel, 2015; Trivedi et al., 2015, 2017)

\subsection{Conclusiones}

Los desafíos actuales, entre los que se encuentra la creciente demanda mundial de alimentos y la necesidad de preservar la salud del suelo, hacen indispensable modificar la forma de producción agrícola. El paquete tecnológico conocido como agricultura de conservación (siembra directa, cultivos de cobertura y la intensificación y diversificación de los cultivos, manejo integral de plagas y fertilización adecuada) parece constituir una respuesta válida para esta demanda. La siembra directa (SD) es sólo el primer paso.

Finalmente debemos remarcar la necesidad de identificar la importancia de los diferentes grupos microbianos con el fin de poder incrementar los servicios ecosistémicos. Además, conocer las funciones de los microorganismos en cada fracción de agregados mejorará nuestro conocimiento sobre la estructura y funcionamiento del suelo y proveerá los datos empíricos necesarios para desarrollar modelos matemáticos más certeros a la hora de predecir la actividad global del suelo (Weil, 2001).

\subsection{Referencias bibliográficas}

Acosta-Martínez, V., Dowd, S., Sun, Y., Allen, V. (2008). Tag-encoded pyrosequencing analysis of bacterial diversity in a single soil type as affected by management and land use. Soil Biol. Biochem, 40, 2762-2770.

Albertengo, J., Belloso, C., Giraudo, M.B., Peiretti, R., Permingeat, H., Wall, L. (2013). 15 Conservation Agriculture in Argentina. Conserv. Agric. Glob. Prospects Chall. 352. 
Allison, S.D., Weintraub, M.N., Gartner, T.B., Waldrop, M.P., (2010). Evolutionaryeconomic principles as regulators of soil enzyme production and ecosystem function, in: Soil Enzymology. Springer, 229-243.

Alvarez, R., Steinbach, H.S., (2009). A review of the effects of tillage systems on some soil physical properties, water content, nitrate availability and crops yield in the Argentine Pampas. Soil Tillage Res. 104, 1-15.

Bach, E.M., Hofmockel, K.S., (2015). A time for every season: soil aggregate turnover stimulates decomposition and reduces carbon loss in grasslands managed for bioenergy. GCB Bioenergy n/a-n/a. https://doi.org/10.1111/gcbb.12267

Balesdent, J., Chenu, C., Balabane, M., (2000). Relationship of soil organic matter dynamics to physical protection and tillage. Soil Tillage Res. 53, 215-230.

Beare, M.H., Hendrix, P.F., Coleman, D.C., (1994). Water-stable aggregates and organic matter fractions in conventional-and no-tillage soils. Soil Sci. Soc. Am. J. 58, 777-786.

Bronick, C.J., Lal, R. (2005). Soil structure and management: a review. Geoderma 124, 3 22.

Christensen, B.T. (2001). Physical fractionation of soil and structural and functional complexity in organic matter turnover. Eur. J. Soil Sci. 52, 345-353.

Constancias, F., Prévost-Bouré, N.C., Terrat, S., Aussems, S., Nowak, V., Guillemin, J.-P., Bonnotte, A., Biju-Duval, L., Navel, A., Martins, J.M., others, (2014). Microscale evidence for a high decrease of soil bacterial density and diversity by cropping. Agron. Sustain. Dev. $34,831-840$.

Crowther, T.W., Todd-Brown, K.E.O., Rowe, C.W., Wieder, W.R., Carey, J.C., Machmuller, M.B., Snoek, B.L., Fang, S., Zhou, G., Allison, S.D., others, (2016). Quantifying global soil carbon losses in response to warming. Nature 540, 104-108.

Dai, J., Hu, J., Zhu, A., Lin, X., (2017). No-tillage with half-amount residue retention enhances microbial functional diversity, enzyme activity and glomalin-related soil protein content within soil aggregates. Soil Use Manag.

Davinic, M., Fultz, L.M., Acosta-Martinez, V., Calderón, F.J., Cox, S.B., Dowd, S.E., Allen, V.G., Zak, J.C., Moore-Kucera, J., (2012). Pyrosequencing and mid-infrared spectroscopy reveal distinct aggregate stratification of soil bacterial communities and organic matter composition. Soil Biol. Biochem. 46, 63-72.

Derpsch, R., Friedrich, T., Kassam, A., Li, H., (2010). Current status of adoption of no-till farming in the world and some of its main benefits. Int. J. Agric. Biol. Eng. 3, 1-25.

Díaz-Zorita, M., Duarte, G.A., Grove, J.H., (2002).A review of no-till systems and soil management for sustainable crop production in the subhumid and semiarid Pampas of Argentina. Soil Tillage Res. 65, 1-18. https://doi.org/10.1016/S0167-1987(01)00274-4

Dıaz-Zorita, M., Perfect, E., Grove, J.H., (2002). Disruptive methods for assessing soil structure. Soil Tillage Res. 64, 3-22.

Ettema, C.H., Wardle, D.A. (2002). Spatial soil ecology. Trends Ecol. Evol. 17, 177-183.

Feng, Y., Motta, A.C., Reeves, D.W., Burmester, C.H., Van Santen, E., Osborne, J.A. (2003). Soil microbial communities under conventional-till and no-till continuous cotton systems. Soil Biol. Biochem. 35, 1693-1703. 
Foley, Z., (2011). Solutions for a cultivated planet. Nature 478, 337-342.

Franzluebbers, A.J., Arshad, M.A. (1996). Soil organic matter pools during early adoption of conservation tillage in northwestern Canada. Soil Sci. Soc. Am. J. 60, 1422-1427.

Frene, J. P., (2017). Estudio fisiológico del suelo bajo diferentes tratamientos agrícolas. Tesis de Doctorado en Ciencia y Tecnología. Universidad Nacional de Quilmes, 212

Gomez, E., Garland, J.L., (2012). Effects of tillage and fertilization on physiological profiles of soil microbial communities.Appl. Soil Ecol., Microorganisms and the Sustainable Management of Soil 61, 327-332. https://doi.org/10.1016/j.apsoil.2011.10.008

Gupta, V., Germida, J. J. (1988). Distribution of microbial biomass and its activity in different soil aggregate size classes as affected by cultivation. Soil Biol. Biochem. 20, 777786.

Hattori, T., (1988). Soil aggregates as microhabitats of microorganisms. Rep. Inst. Agric. Res. Tohoku Univ. 37, 23-36.

Helgason, B.L., Walley, F.L., Germida, J.J., (2010). No-till soil management increases microbial biomass and alters community profiles in soil aggregates. Appl. Soil Ecol. 46, 390-397.

Jangid, K., Williams, M.A., Franzluebbers, A.J., Sanderlin, J.S., Reeves, J.H., Jenkins, M.B., Endale, D.M., Coleman, D.C., Whitman, W.B., (2008). Relative impacts of land-use, management intensity and fertilization upon soil microbial community structure in agricultural systems. Soil Biol. Biochem. 40, 2843-2853.

Jiang, X., Wright, A.L., Wang, J., Li, Z., (2011a). Long-term tillage effects on the distribution patterns of microbial biomass and activities within soil aggregates. Catena 87, 276-280.

Jiang, X., Wright, A.L., Wang, X., Liang, F., (2011b). Tillage-induced changes in fungal and bacterial biomass associated with soil aggregates: a long-term field study in a subtropical rice soil in China. Appl. Soil Ecol. 48, 168-173.

Kandeler, E., Luxhøi, J., Tscherko, D., Magid, J., (1999a). Xylanase, invertase and protease at the soil-litter interface of a loamy sand. Soil Biol. Biochem. 31, 1171-1179.

Kandeler, E., Palli, S., Stemmer, M., Gerzabek, M.H., (1999b). Tillage changes microbial biomass and enzyme activities in particle-size fractions of a Haplic Chernozem. Soil Biol. Biochem. 31, 1253-1264.

Kandeler, E., Stemmer, M., Klimanek, E.-M., (1999c). Response of soil microbial biomass, urease and xylanase within particle size fractions to long-term soil management. Soil Biol. Biochem. 31, 261-273. https://doi.org/10.1016/S0038-0717(98)00115-1

Kandeler, E., Tscherko, D., Spiegel, H., (1999). Long-term monitoring of microbial biomass, $\mathrm{N}$ mineralisation and enzyme activities of a Chernozem under different tillage management. Biol. Fertil. Soils 28, 343-351.

Kong, A.Y., Scow, K.M., Córdova-Kreylos, A.L., Holmes, W.E., Six, J., (2011). Microbial community composition and carbon cycling within soil microenvironments of conventional, low-input, and organic cropping systems. Soil Biol. Biochem. 43, 0e30. 
Lagomarsino, A., Grego, S., Kandeler, E. (2012). Soil organic carbon distribution drives microbial activity and functional diversity in particle and aggregate-size fractions. Pedobiologia 55, 101-110.

Lal, R., (2015). Restoring Soil Quality to Mitigate Soil Degradation. Sustainability 7, 58755895. https://doi.org/10.3390/su7055875

Lal, R., (2004). Soil carbon sequestration impacts on global climate change and food security. Science 304, 1623-1627.

Lal, R., (1998). Soil erosion impact on agronomic productivity and environment quality. Crit. Rev. Plant Sci. 17, 319-464.

Leff, J.W., Jones, S.E., Prober, S.M., Barberán, A., Borer, E.T., Firn, J.L., Harpole, W.S., Hobbie, S.E., Hofmockel, K.S., Knops, J.M.H., McCulley, R.L., Pierre, K.L., Risch, A.C., Seabloom, E.W., Schütz, M., Steenbock, C., Stevens, C.J., Fierer, N., (2015). Consistent responses of soil microbial communities to elevated nutrient inputs in grasslands across the globe. Proc. Natl. Acad. Sci. 112, 10967-10972. https://doi.org/10.1073/pnas.1508382112

Lladó, S., Baldrian, P. (2017). Community-level physiological profiling analyses show potential to identify the copiotrophic bacteria present in soil environments. PloS One 12, e0171638.

Madsen, E.L., (2005). Identifying microorganisms responsible for ecologically significant biogeochemical processes. Nat. Rev. Microbiol. 3, 439-446.

Mbuthia, Acosta-MArtinez, DeBruyn, Schaeffer, Tyler, Odoi, Mpheshea, Walker, Eash, (2015). Long term tillage, cover crop, and fertilization effects on microbial community structure, activity: Implications for siol quality. Soil Biol. Biochem. 89, 24-34.

Milenium Ecosystem Assesment. (2005). Ecosystems and human well-being: Synthesis. Island Press. Washington. DC.

Mummey, D., Holben, W., Six, J., Stahl, P., (2006). Spatial stratification of soil bacterial populations in aggregates of diverse soils. Microb. Ecol. 51, 404-411.

Nannipieri, P., Ascher, J., Ceccherini, M., Landi, L., Pietramellara, G., Renella, G., (2003). Microbial diversity and soil functions. Eur. J. Soil Sci. 54, 655-670.

Nelson, M.B., Martiny, A.C., Martiny, J.B., (2016). Global biogeography of microbial nitrogen-cycling traits in soil. Proc. Natl. Acad. Sci. 113, 8033-8040.

Neumann, D., Heuer, A., Hemkemeyer, M., Martens, R., Tebbe, C.C., (2013). Response of microbial communities to long-term fertilization depends on their microhabitat. FEMS Microbiol. Ecol. 86, 71-84.

Pandey, D., Agrawal, M., Bohra, J.S., (2014). Effects of conventional tillage and no tillage permutations on extracellular soil enzyme activities and microbial biomass under rice cultivation. Soil Tillage Res. 136, 51-60.

Phatak, S.C., Reed, R., Fussell, W., Lewis, W.J., Harris, G.H., (1999). Crimson clover cotton relay cropping with conservation tillage system, in: Proceedings of the 22nd Annual Southern Conservation Tillage Conference for Sustainable Agriculture, Tifton, GA. pp. 184188. 
Philippot, L., Bru, D., Saby, N., Čuhel, J., Arrouays, D., Šimek, M., Hallin, S., (2009). Spatial patterns of bacterial taxa in nature reflect ecological traits of deep branches of the 16S rRNA bacterial tree. Environ. Microbiol. 11, 3096-3104.

Pittelkow, C.M., Linquist, B.A., Lundy, M.E., Liang, X., Van Groenigen, K.J., Lee, J., Van Gestel, N., Six, J., Venterea, R.T., Van Kessel, C., (2015). When does no-till yield more? A global meta-analysis. Field Crops Res. 183, 156-168.

Qin, S., Hu, C., He, X., Dong, W., Cui, J., Wang, Y., (2010). Soil organic carbon, nutrients and relevant enzyme activities in particle-size fractions under conservational versus traditional agricultural management. Appl. Soil Ecol. 45, 152-159.

Roldán, A., Salinas-García, J.R., Alguacil, M.M., Caravaca, F., (2005). Changes in soil enzyme activity, fertility, aggregation and $C$ sequestration mediated by conservation tillage practices and water regime in a maize field. Appl. Soil Ecol. 30, 11-20.

Sessitsch, A., Weilharter, A., Gerzabek, M.H., Kirchmann, H., Kandeler, E., (2001). Microbial population structures in soil particle size fractions of a long-term fertilizer field experiment. Appl. Environ. Microbiol. 67, 4215-4224.

Silva, A.P., Babujia, L.C., Matsumoto, L.S., Guimarães, M.F., Hungria, M., (2013). Bacterial diversity under different tillage and crop rotation systems in an Oxisol of southern Brazil. Open Agric. J. 7, 40-47.

Simmons, B.L., Coleman, D.C., (2008). Microbial community response to transition from conventional to conservation tillage in cotton fields. Appl. Soil Ecol. 40, 518-528.

Six, J., Elliott, E.T., Paustian, K., (1999). Aggregate and soil organic matter dynamics under conventional and no-tillage systems. Soil Sci. Soc. Am. J. 63, 1350-1358.

Six, J., Feller, C., Denef, K., Ogle, S., Sa, J.C.D.M., Albrecht, A., (2002). Soil organic matter, biota and aggregation in temperate and tropical soils-Effects of no-tillage. Agronomie 22, 755-775.

Six, J., Frey, S.D., Thiet, R.K., Batten, K.M., (2006). Bacterial and fungal contributions to carbon sequestration in agroecosystems. Soil Sci. Soc. Am. J. 70, 555-569.

Stemmer, M., Gerzabek, M.H., Kandeler, E., (1998). Organic matter and enzyme activity in particle-size fractions of soils obtained after low-energy sonication. Soil Biol. Biochem. 30, 9-17.

Tate, K.R., Scott, N.A., Parshotam, A., Brown, L., Wilde, R.H., Giltrap, D.J., Trustrum, N.A., Gomez, B., Ross, D.J., (2000). A multi-scale analysis of a terrestrial carbon budget: is New Zealand a source or sink of carbon? Agric. Ecosyst. Environ. 82, 229-246.

Tiemann, L.K., Grandy, A.S., (2015). Mechanisms of soil carbon accrual and storage in bioenergy cropping systems. Gcb Bioenergy 7, 161-174.

Tiemann, L.K., Grandy, A.S., Atkinson, E.E., Marin-Spiotta, E., McDaniel, M.D., (2015). Crop rotational diversity enhances belowground communities and functions in an agroecosystem. Ecol. Lett. 18, 761-771.

Triplett, G.B., Dick, W.A., (2008). No-tillage crop production: a revolution in agriculture! Agron. J. 100, S-153.

Trivedi, P., Delgado-Baquerizo, M., Jeffries, T.C., Trivedi, C., Anderson, I.C., McNee, M., Flower, K., Pal Singh, B., Minkey, D., Singh, B.K., (2017). Soil aggregation and associated 
microbial communities modify the impact of agricultural management on carbon content. Environ. Microbiol.

Trivedi, P., Rochester, I.J., Trivedi, C., Van Nostrand, J.D., Zhou, J., Karunaratne, S., Anderson, I.C., Singh, B.K., (2015). Soil aggregate size mediates the impacts of cropping regimes on soil carbon and microbial communities. Soil Biol. Biochem. 91, 169-181.

Vos, M., Wolf, A.B., Jennings, S.J., Kowalchuk, G.A., (2013). Micro-scale determinants of bacterial diversity in soil. FEMS Microbiol. Rev. 37, 936-954.

Weil, R.W., (2001). Soil management for sustainable intensification: Some guidelines. ASA Spec. Publ. 64, 145-154.

West, T.O., Post, W.M., (2002). Soil organic carbon sequestration rates by tillage and crop rotation. Soil Sci. Soc. Am. J. 66, 1930-1946.

Wortmann, C.S., Quincke, J.A., Drijber, R.A., Mamo, M., Franti, T., (2008). Soil microbial community change and recovery after one-time tillage of continuous no-till. Agron. J. 100, $1681-1686$.

Zhang, B., Li, Y., Ren, T., Tian, Z., Wang, G., He, X., Tian, C., (2014). Short-term effect of tillage and crop rotation on microbial community structure and enzyme activities of a clay loam soil. Biol. Fertil. Soils 50, 1077-1085.

Zhang, Q., Liang, G., Zhou, W., Sun, J., Wang, X., He, P., (2016). Fatty-Acid Profiles and Enzyme Activities in Soil Particle-Size Fractions under Long-Term Fertilization. Soil Sci. Soc. Am. J. 80, 97-111.

Zhao, X., Liu, S.-L., Pu, C., Zhang, X.-Q., Xue, J.-F., Ren, Y.-X., Zhao, X.-L., Chen, F., Lal, R., Zhang, H.-L., (2017). Crop yields under no-till farming in China: A meta-analysis. Eur. J. Agron. 84, 67-75. https://doi.org/10.1016/j.eja.2016.11.009 